\title{
Work-related medical rehabilitation in patients with musculoskeletal disorders: the protocol of a propensity score matched effectiveness study (EVA-WMR, DRKS00009780)
}

\author{
Silke Neuderth ${ }^{1}$, Betje Schwarz ${ }^{2}$, Christian Gerlich ${ }^{3}$, Michael Schuler ${ }^{3^{*}}$ (D, Miriam Markus ${ }^{2}$ and Matthias Bethge ${ }^{2}$
}

\begin{abstract}
Background: Musculoskeletal disorders are one of the most important causes of work disability. Various rehabilitation services and return-to-work programs have been developed in order to reduce sickness absence and increase sustainable return-to-work. As the effects of conventional medical rehabilitation programs on sickness absence duration were shown to be slight, work-related medical rehabilitation programs have been developed and tested. While such studies proved the efficacy of work-related medical rehabilitation compared with conventional medical rehabilitation in well-conducted randomized controlled trials, its effectiveness under real-life conditions has yet to be proved.

Methods/Design: The cohort study will be performed under real-life conditions with two parallel groups. Participants will receive either a conventional or a work-related medical rehabilitation program. Propensity score matching will be used to identify controls that are comparable to treated work-related medical rehabilitation patients. Over a period of three months, about 18,000 insured patients with permission to undergo a musculoskeletal rehabilitation program will be contacted. Of these, 15,000 will receive a conventional and 3,000 a work-related medical rehabilitation. We expect a participation rate of $40 \%$ at baseline. Patients will be aged 18 to 65 years and have chronic musculoskeletal disorders, usually back pain. The control group will receive a conventional medical rehabilitation program without any explicit focus on work, work ability and return to work in diagnostics and therapy. The intervention group will receive a work-related medical rehabilitation program that in addition to common rehabilitation treatments contains 11 to $25 \mathrm{~h}$ of work-related treatment modules. Follow-up data will be assessed three and ten months after patients' discharge from the rehabilitation center. Additionally, department characteristics will be assessed and administrative data records used. The primary outcomes are sick leave duration, stable return to work and subjective work ability. Secondary outcomes cover several dimensions of health, functioning and coping strategies.
\end{abstract}

Discussion: This study will determine the relative effectiveness of a complex, newly implemented work-related rehabilitation strategy for patients with musculoskeletal disorders.

Trial registration: German Clinical Trials Register (DRKS00009780, February 10, 2016).

Keywords: Work-related medical rehabilitation, Effectiveness, Work ability, Return to work, Propensity score matching

\footnotetext{
*Correspondence: m.schuler@uni-wuerzburg.de

${ }^{3}$ Department of Medical Psychology and Psychotherapy, Medical Sociology

and Rehabilitation Sciences, University of Würzburg, Klinikstraße 3, Würzburg

97070, Germany

Full list of author information is available at the end of the article
}

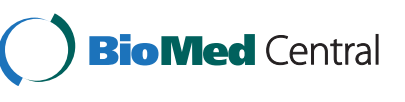

(c) 2016 The Author(s). Open Access This article is distributed under the terms of the Creative Commons Attribution 4.0 International License (http://creativecommons.org/licenses/by/4.0/), which permits unrestricted use, distribution, and reproduction in any medium, provided you give appropriate credit to the original author(s) and the source, provide a link to the Creative Commons license, and indicate if changes were made. The Creative Commons Public Domain Dedication waiver (http://creativecommons.org/publicdomain/zero/1.0/) applies to the data made available in this article, unless otherwise stated. 


\section{Background}

About 35 million people aged 15 to $64(11.0 \%)$ in the European Union are limited in the work they can do because of a long-standing health problem or a basic activity difficulty [1]. As musculoskeletal disorders are still one of the most important causes of work disability, various rehabilitation services and return-to-work programs have been developed and implemented in order to battle the consequences of musculoskeletal disorders for the opportunity to participate in working life [2]. As shown by systematic reviews, such programs and interventions indeed may significantly reduce sickness absence and increase sustainable return-to-work, especially if they include work-related elements [3-5].

In Germany, rehabilitation programs for musculoskeletal disorders are usually provided as three-week in- or outpatient interventions under the umbrella of the German Pension Insurance (GPI). The treatment is multiprofessional and follows evidence-based therapy recommendations [6]. However, change in sickness absence duration was shown to be slight in meta-analyses of observational studies $[7,8]$. One randomized controlled trial indicated no effect on sickness absence duration [9]. Moreover, several studies revealed that patients with severe restrictions of work ability (e.g. long-term sick leave, poor return-to work expectation) have a considerable risk of not returning to work despite participating in a conventional medical rehabilitation (CMR) program [10, 11]. Therefore, work-related medical rehabilitation (WMR) programs have been developed and tested in recent years $[10,12]$.

These programs were designed to particularly support patients with severe restrictions in terms of ability to work. WMR programs regularly comprise a diagnostic assessment that compares job demands and patients' work capacity and offer therapeutic interventions such as work hardening and work-related functional capacity training, work-related psychosocial groups and intensified social counseling [13-16]. A meta-analytic synthesis of randomized controlled trials that compared WMR and CMR programs in Germany provides robust evidence that in patients with musculoskeletal disorders WMR programs achieve higher rates of sustainable return-to-work and reduce sickness absence after one year. WMR participants had 2.4 times higher odds of sustainable return-to-work after one year and also reduced sickness absence [17].

To support nationwide implementation of such programs and to improve rehabilitation outcomes, the GPI developed a WMR guideline. This guideline defines inclusion criteria for WMR (e.g. long-term sick leave, poor self-rated return-to-work expectation, unemployment) and important diagnostic and therapeutic measures in WMR (see Treatment section for a more detailed description of WMR) [13, 18]. The implementation of the WMR guideline was evaluated in patients with musculoskeletal disorders in seven rehabilitation centers. The results showed that the implementation was challenging but feasible. Moreover, the observational study showed a significant reduction of sick leave for WMR participants, and more work-related interventions predicted shorter sickness absence at the three-month follow-up [19]. However, findings also indicated that allocation and treatment decisions (WMR vs. CMR) only partly followed the guideline recommendations [20].

While studies proved the efficacy of WMR compared with CMR in well-conducted randomized controlled trials with high treatment fidelity and carefully selected patients, the effectiveness of WMR under real-life conditions has yet to be proved. Interventions that work in efficacy studies may not necessarily also do well in realworld applications [21, 22]. Though reliable efficacy studies are a necessary condition for evidence-based practice, patients and other stakeholders may be most interested in the effectiveness of real-world services. While a randomized controlled trial is the gold standard and the most robust way to prevent allocation bias in efficacy studies, other designs and methods may be needed to evaluate the effectiveness of an intervention, especially, as in the case of WMR, where nationwide dissemination is completed and programs and allocation procedures are established. Nevertheless, as the WMR guideline clearly describes the patients in need and proposes several screenings to identify these patients [11], allocation bias is a severe challenge in determining an unbiased treatment effect estimate. One proper evaluation design in this case is the use of observational data and a subsequent propensity score matched analysis to control for confounding. We therefore designed a cohort study to analyze the relative effectiveness of WMR compared with CMR. As current allocation procedures regarding WMR seem to be far from perfect and WMR and CMR patients have a considerable overlap, a propensity matched comparison was chosen to address allocation bias. We hypothesize that WMR reduces sickness absence and improves sustainable return-to-work and work ability ten months after rehabilitation (primary outcomes) compared with CMR. Moreover, we expect to see evidence of the superior effectiveness of WMR regarding several secondary outcomes. The study protocol has been prepared according to the SPIRIT checklist (Standard Protocol Items: Recommendations for Interventional Trials) [23].

\section{Methods \\ Study design}

The study is a cohort study under real-life conditions with two parallel groups. Participants will either receive a WMR or a CMR program in one of the 256 approved rehabilitation departments. The allocation ratio is determined by the actual allocation under real-life conditions of rehabilitation service provision in Germany. The current ratio of WMR 
to CMR patients is approximately one to five. The investigators will not have any influence on allocation decisions. Current utilization of WMR is still considerably below the estimated number of patients who need it. Therefore, comparable controls are available who receive a CMR, though their severe restrictions in terms of work ability would also justify participation in a WMR program. Although the researchers have no influence on the allocation procedure they will have the opportunity to model treatment allocation by observed data. Thus propensity score matching will be used to identify controls that are comparable to treated WMR patients and to estimate the unbiased effects of the relative effectiveness of WMR compared with CMR. As randomized controlled trials confirmed the relative efficacy of WMR in trials with high treatment fidelity and carefully selected patients, and implementation of WMR is completed, we assume the superiority of WMR.

Baseline data will be assessed after approval of the rehabilitation program but before the patients begin their rehabilitation. Follow-up data will be assessed three and ten months after their discharge from the rehabilitation center. Moreover, administrative data records will be used. Additionally, department characteristics will be assessed by a departmental survey. No one will be blinded before, during or after the trial.

\section{Study setting}

All included rehabilitation centers are located in Germany. Rehabilitation services may be provided as inpatient or outpatient programs. Most of the departments are inpatient centers. There are some outpatient departments that are mainly located in major cities. Participation in a rehabilitation program was approved by the Federal GPI. In both groups, interventions will be performed by rehabilitation physicians, psychologists, physiotherapists, sports therapists, social workers, occupational therapists and other health professionals.

The duration of the rehabilitation program is initially determined by the Federal GPI (usually about three weeks). The rehabilitation center and the patient may arrange to extend the program. By request the patient may stop the rehabilitation program ahead of schedule.

\section{Treatment}

\section{Control}

Participants of the control group will receive a CMR program according to current treatment standards and guidelines for the rehabilitation of musculoskeletal disorders. CMR programs last approximately three weeks. The daily quantum of therapy amounts to three or four hours. Following a multimodal approach, CMR programs include sports and exercise therapy, physiotherapy, occupational therapy, massage and other physical therapies, social and psychological counseling, patient education, pain management and relaxation training. CMR programs focus on the functional limitations of the musculoskeletal system and aim to restore physical abilities to promote participation in work and daily living. However, in contrast to WMR programs, they do not integrate an explicit focus on work, work ability and return to work in diagnostics and therapy.

\section{Intervention}

Participants of the intervention group will receive a WMR program according to the guideline for $\operatorname{WMR}[13,18]$ as well as to the current treatment standards and guidelines for the rehabilitation of musculoskeletal disorders. WMR programs last approximately 2.4 days longer than CMR programs since they contain 11 to $25 \mathrm{~h}$ of work-related treatment modules [17]. Like CMR programs WMR programs follow a multimodal approach that comprises sports and exercise therapy, physiotherapy, occupational therapy, massage and other physical therapies, social and psychological counseling, patient education, pain management and relaxation training. However, WMR programs more explicitly focus on work, work ability and return to work. Thus, they include intensified work-related diagnostics as well as work-related functional capacity training, work-related psychosocial groups and intensified social counseling. Intensified work-related diagnostics identify individual needs by comparing work-related physical and psychosocial functional capacity with the patient's job demands. Assessment of functional capacity is performed by means of a short functional capacity evaluation and questionnaires. Demands will be assessed by job analysis. Matching of capacity and demands is supported by standardized assessments [24-26]. Work-related functional capacity training exercises specific movements and postures according to individual workplace conditions. Work-related psychosocial groups deal with the mutual dependence of behavioral and emotional health experiences and the workplace environment. Additionally, preventative measures like stress management or conflict management in dealing with psychosocial stressors are taught. Finally, intensified social counseling examines the individual work-life situation and provides socio-legal guidance and advice concerning further assistance within the social security system.

\section{Participants}

Patients are aged 18 to 65 years and have chronic musculoskeletal disorders, usually back pain. They have approval for rehabilitation either in an own rehabilitation institution of the Federal GPI or an institution that is leadingly occupied by the Federal GPI. Patients have requested rehabilitation because of health-related restrictions of work ability. Need for rehabilitation was acknowledged by a registered doctor and approved by the Federal GPI. 
Allocation to CMR and WMR is primarily decided by the socio-medical service of the Federal GPI. The sociomedical service may consider the findings of standardized screening which is usually part of the application documents and estimates the risk of not returning to work [11].

\section{Sample size estimation}

We expect to find small mean differences between WMR and CMR in primary outcomes. For three months, of about 18,000 insured patients with permission for a musculoskeletal rehabilitation program 15,000 will receive CMR and 3,000 will receive WMR. We expect a participation rate of $40 \%$, i.e. 7,200 patients $(6,000$ CMR, 1,200 WMR) at baseline. At the 10-month follow-up, we expect a dropout rate of $25 \%$, i.e. 900 patients with WMR and 4,500 CMR left. Using a one-to-one match without replacement, 1,800 patients (900 WMR, $900 \mathrm{CMR}$ ) will be analyzed. Assuming a number of clusters (departments) $k=256$ (with 176 departments providing CMR and 80 departments providing additionally WMR), a cluster size of $m=7.0$, an intracluster correlation of rho $=0.05$, a power of 0.80 and a Bonferroni corrected $p$-value $=0.016$, an effect of SMD $=0.175$ can be detected (Fig. 1).

\section{Recruitment}

Participants with chronic musculoskeletal complaints whose request for rehabilitation was approved will be contacted by postal mail. Mails will be delivered in three waves at four-week intervals. All persons whose rehabilitation measure was approved within the previous four weeks will be contacted in each wave. The letter contains information about the study with the request to participate and the baseline questionnaire. The questionnaire contains no personal data but a unique study identifier. No personal data (e.g. name and address) will be submitted or published by the Federal GPI. One week after the initial postal contact a reminder will be sent to all potential participants. All participants will be thanked and reminded to participate if they have not yet done so. By this method, we expect to increase participant enrolment in order to reach our estimated target sample size and to strengthen external validity. Informed consent is assumed if patients complete their questionnaire and send the questionnaire to the research team. Additionally, participants will be asked to give permission to use administrative data from their pension insurance accounts and to link these data with the questionnaire data.

At the three- and ten-month follow-up participants who complete the baseline questionnaire will receive follow-up questionnaires from the Federal GPI (Table 1). Three weeks later the questionnaire will be sent again with a reminder to all participants who have not completed the questionnaire yet.

\section{Data management}

Questionnaires will be scanned and verified by an electronic data capture system and exported to statistical software packages for further analysis. Scanning and verifying

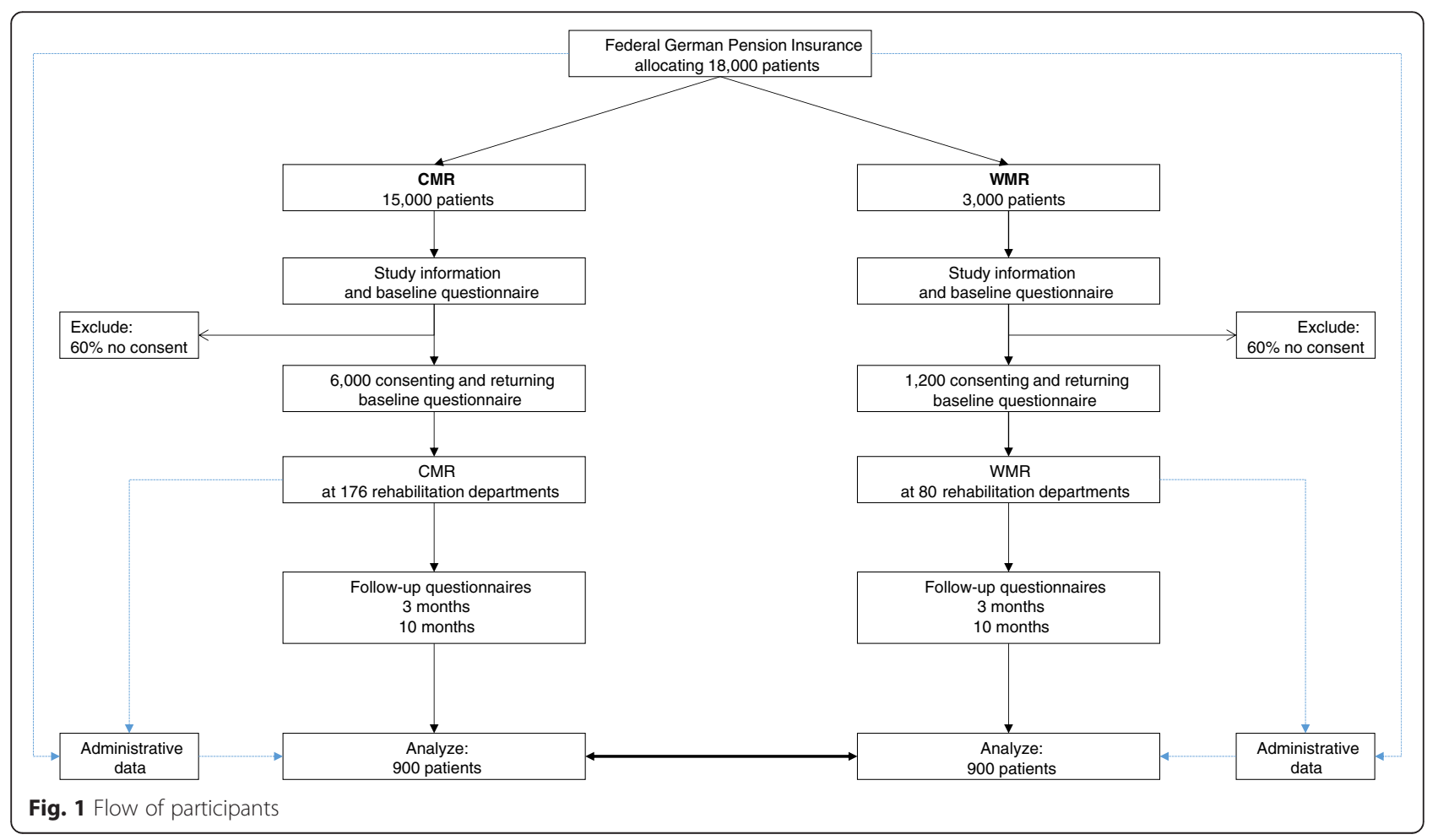


Table 1 Schedule of enrolment, interventions, and assessments

\begin{tabular}{|c|c|c|c|c|c|c|}
\hline \multirow[b]{2}{*}{ TIMEPOINT } & \multicolumn{6}{|c|}{ STUDY PERIOD } \\
\hline & Allocation & $\begin{array}{l}\text { Pre- } \\
\text { intervention }\end{array}$ & $\begin{array}{l}\text { Beginning } \\
\text { of the } \\
\text { intervention }\end{array}$ & $\begin{array}{l}\text { End of the } \\
\text { intervention }\end{array}$ & $\begin{array}{l}\text { 3-month } \\
\text { follow-up }\end{array}$ & $\begin{array}{l}\text { 10-month } \\
\text { follow-up }\end{array}$ \\
\hline \multicolumn{7}{|l|}{ ENROLMENT } \\
\hline Information letter & & $\mathrm{X}$ & & & & \\
\hline Allocation (under real-life conditions) & $\mathrm{X}$ & & & & & \\
\hline \multicolumn{7}{|l|}{ INTERVENTIONS } \\
\hline \multicolumn{7}{|l|}{ Conventional medical rehabilitation } \\
\hline \multicolumn{7}{|l|}{ Work-related medical rehabilitation } \\
\hline \multicolumn{7}{|l|}{ ASSESSMENTS } \\
\hline Patient baseline survey & & $\mathrm{X}$ & & & & \\
\hline Patient 3-month follow-up & & & & & $\mathrm{X}$ & \\
\hline Patient 10-month follow-up & & & & & & $\mathrm{X}$ \\
\hline Department survey & & & & & $\mathrm{X}$ & \\
\hline $\begin{array}{l}\text { Administrative data on received } \\
\text { therapeutic interventions }\end{array}$ & & & & & & $\mathrm{X}$ \\
\hline $\begin{array}{l}\text { Administrative data on income and welfare } \\
\text { benefits }\end{array}$ & & $\mathrm{X}$ & & & & $\mathrm{X}$ \\
\hline
\end{tabular}

will be done by trained research assistants. They will check electronically processed data item by item and compare imported data with the original questionnaire data. Administrative data will be extracted by the Federal GPI. Personal data will be removed and replaced by the unique study identifier. Data will be transferred to the principal investigator (MB). Finally, questionnaire and administrative data can be linked by the unique study identifier.

Data management will be done by the authors of the protocol. Data access is limited to the authors and the research assistants of the research team.

\section{Outcomes and other measures}

This study will assess three primary outcomes as well as secondary outcomes, moderator variables and variables for propensity score matching. Outcomes and other measures will be assessed with patient questionnaires or will be extracted from administrative records (i.e. individual pension insurance accounts) provided by the Federal GPI. A complete list of all measured constructs, measurement points and expected scaling is shown in Table 2.

\section{Primary outcomes}

The primary outcomes of this study are sick leave duration, stable return to work, and subjective work ability. Evaluation of the effectiveness of WMR relates to the 10-month follow-up. These three outcomes will also be assessed at the three-month follow-up and if appropriate at baseline.

To assess the duration of sick leave, the participants will be asked to report the number of weeks they have been off work for health reasons since discharge from the rehabilitation center. At baseline this question is related to the last 12 months. Stable return to work has been defined in accordance with Kuijer and colleagues [27] as a 
Table 2 Measures, assessment, expected scaling and measurement occasions

\begin{tabular}{|c|c|c|c|c|c|}
\hline Outcome & Source and reference & Scaling & Baseline & 3-month follow-up & 10-month follow-up \\
\hline \multicolumn{6}{|l|}{ Primary outcomes } \\
\hline Sick leave duration in weeks & Own development & metric & $x$ & $x$ & $x$ \\
\hline Stable return to work & Own development & binary & $x$ & $x$ & $x$ \\
\hline Subjective work ability & WAS [28] & metric & $x$ & $x$ & $x$ \\
\hline \multicolumn{6}{|l|}{ Secondary outcomes } \\
\hline General health & COPSOQ (1 item) $[30,31]$ & metric & $x$ & $x$ & $x$ \\
\hline Physical functioning & IRES-24 [32] & metric & $x$ & $x$ & $x$ \\
\hline Depression & PHQ-2 [33] & metric/binary & $x$ & $x$ & $x$ \\
\hline Anxiety & GAD-2 [34] & metric/binary & $x$ & $x$ & $x$ \\
\hline Pain intensity & CPQ $[35,36]$ & metric & $x$ & $x$ & $x$ \\
\hline Pain disability & CPQ $[35,36]$ & metric & $x$ & $x$ & $x$ \\
\hline Fear of movement & FABQ $[37,38]$ & metric & $x$ & $x$ & $x$ \\
\hline Physical activity & Modified Godin-Scale [39] & metric & $x$ & $x$ & $x$ \\
\hline Medication use & Own development & nominal & $x$ & $x$ & $x$ \\
\hline Self-management skills & heiQ [40] & metric & $x$ & $x$ & $x$ \\
\hline Employment & Own development & binary & $x$ & $x$ & $x$ \\
\hline Current sickness absence & Own development & binary & $x$ & $x$ & $x$ \\
\hline Subjective prognosis of employment status & SPE [41] & metric/binary & $x$ & $x$ & $x$ \\
\hline Implementation of work-related interventions & [19] & metric & & $x$ & \\
\hline Consistency of the work-related strategy & [19] & metric & & $x$ & \\
\hline Work-related benefit & [19] & metric & & $x$ & \\
\hline Treatment satisfaction & CSQ-8 [42] & metric & & $x$ & \\
\hline Income and welfare benefits & GPI accounts & metric & $x$ & & $x$ \\
\hline Therapeutic interventions & GPI accounts & metric & & & $x$ \\
\hline Department characteristics & Department survey & metric/nominal & & & $x$ \\
\hline \multicolumn{6}{|l|}{ Other measures } \\
\hline Risk scores for not returning to work & SIMBO [11], WS [12] & & $x$ & & \\
\hline Somatization & SCL-90-R [43] & metric & $x$ & & \\
\hline Pain generalization & Own development & metric & $x$ & & \\
\hline Psychosocial stress & [44] & metric & $x$ & & \\
\hline Socio-demographic data & Own development & metric/nominal & $x$ & & \\
\hline Size of company & [45] & nominal & $x$ & & \\
\hline Physical job demands & [46] & metric & $x$ & & \\
\hline Effort-reward imbalance & [47] & metric & $x$ & & \\
\hline Overcommitment & [47] & metric & $x$ & & \\
\hline Support by co-workers and supervisors & Own development & metric & $x$ & & \\
\hline
\end{tabular}

Notes: SCL-90-R Symptom Check-List-90-R, SIMBO Screening-Instrument zur Feststellung des Bedarfs an medizinisch-beruflich orientierten Maßnahmen in der medizinischen Rehabilitation (Screening to assess the need for work-related medical rehabilitation), COPSOQ Copenhagen Psychosocial Questionnaire, CPQ Chronic Pain Grade Questionnaire, CSQ-8 Client Satisfaction Questionnaire, FABQ Fear-Avoidance Beliefs Questionnaire, GAD-2 Generalized Anxiety Disorder Questionnaire, heiQ Health Education Impact Questionnaire, IRES-24 Indikatoren des Reha-Status (Indicators for rehabilitation status), PHQ-2 Patient Health Questionnaire, SPE Subjective prognosis of employment status, WAS Work Ability Score, WS Würzburger Screening

minimum of four weeks of employment without sick leave. Subjective work ability will be assessed by the Work Ability Score (WAS), which is the first item of the Work Ability Index (WAI) [28]. It compares current subjective work ability with the lifetime best. The 11-point scale ranges from zero (complete incapacity to work) to ten (lifetime's best work ability). The WAS is highly correlated with the overall WAI score [29]. 


\section{Secondary outcomes}

Secondary outcomes cover several dimensions of health, functioning and coping strategies (see below) and will be measured at all three measurement points. At the threemonth follow-up, participants will also evaluate how the rehabilitation programs dealt with work-related issues as well as satisfaction with the treatment.

General health One item of the Copenhagen Psychosocial Questionnaire will be used to measure general health. The 11-point scale ranges from zero (worst imaginable health state) to 10 (best imaginable health state) $[30,31]$.

Physical functioning Physical functioning is measured by eight items of the IRES-24 (German: Indikatoren des Reha-Status) [32], a widely used instrument in rehabilitation research in Germany. All items are measured on a five-point scale. Item values are averaged and rescaled to a range from zero to 10 points, with lower values indicating less functioning.

Depression and anxiety The two-item versions of both the Patient Health Questionnaire (PHQ-2) and the Generalized Anxiety Disorder Questionnaire (GAD-2) will be used to assess depression [33] and anxiety [34]. The PHQ-2 uses the first two items of the PHQ-9 ("little interest or pleasure in doing things," "feeling down, depressed or hopeless") while the GAD-2 uses the first two items of the GAD-7 ("feeling nervous, anxious or on edge", "not being able to stop or control worrying"). All items are measured on a four-point scale $(0=$ not at all, $1=$ several days, $2=$ more than half of the days, $3=$ nearly all days). Sum scores for depression and anxiety range from zero to six points. Additionally, we will determine binary outcomes by categorizing values of $>2$ as clinically relevant depressive or anxiety disorder.

Pain intensity and disability Six items from the Graded Chronic Pain Scale will be used to assess pain intensity and pain disability $[35,36]$. Pain intensity items cover current pain and worst pain in the last four weeks and average pain in the last four weeks. Pain disability is related to interference with daily activities, recreational, social and family activities and work activities. All items are scored from zero to 10 points, with higher scores indicating stronger pain or more disability. Items will be averaged and multiplied by 10 . Thus, the composite scores range from zero to 100 points.

Fear of movement Two items of the Fear-Avoidance Beliefs Questionnaire [37] ("My pain was caused by physical activity," "I should not do physical activities which might make my pain worse") will be used to assess fear of movement. Like Kent and colleagues [38], we will use scaling from zero (completely disagree) to 10 (completely agree) instead of the original scaling. A composite score is determined by averaging both item scores.

Physical activity A modified version of the Godin Leisure-Time Exercise Questionnaire [39] will be used to assess how often per week and how long per session patients performed light, moderate and strenuous physical exercise. The total physical activity score in minutes per week will be calculated by multiplying the total number of sessions per week (in each domain) by the minutes per session (in each domain). Additionally, a total sum score will be calculated by multiplying the scores of each domain with the metabolic equivalent of tasks ( $9=$ strenuous, $5=$ moderate and $3=$ light physical exercise).

Medication use Medication used to reduce pain (e.g. Paracetamol), to enhance mood (e.g. Citalopram) or to treat other health complaints will be assessed by three new developed items. Response categories are no, regularly (e.g. daily) and rescue medication.

Self-management skills Self-management skills will be assessed with three items of the scale Skill and technique acquisition of the Health Education Impact Questionnaire [40]. The items are measured on a four-point scale $(1=$ strongly disagree, 2 = disagree, 3 =agree, $4=$ strongly agree). The scale score is the unweighted mean of all items, with higher values indicating higher subjective selfmanagement skills.

Employment To cover participation in working life the employment status (employed vs. unemployed) is asked for. Moreover, we will assess if the patients are on sick leave.

Subjective prognosis of employment status Three items assess if patients believe they will remain at work until retirement, if patients assume that their health will be permanently jeopardized, and if patients intend to submit a request for a disability pension [41]. Items are summarized to reflect a total score ranging from zero to three points. Higher values indicate a more unfavorable outcome. A score of at least two points reflects that permanent work participation is deemed to be unlikely.

Perceived vocational orientation of the rehabilitation program The realization of work-related goals and therapies during rehabilitation will be assessed with a slightly modified version of a previously used set of items from a study that investigated the implementation of the WMR guideline [19]. Participants report on 12 dichotomized items as to whether they received WMR contents throughout their rehabilitation program. Scores are aggregated to a total score ranging from zero to 12 points. This score 
reflects the implementation of the work-related therapies. Additionally, six items assess the perceived diagnostic and therapeutic focus on issues of return to work and work ability, e.g. the experience of a consistent return to work strategy. These items are measured on a five-point scale. Scores will be summed to a total score ranging from zero to 24 points. Finally, the subjective work-related benefit from participating in the rehabilitation program will be assessed by eight items measured on a five-point scale. Scores will be aggregated to a total score ranging from zero to 32 points.

Treatment satisfaction Treatment satisfaction will be assessed using the German version of the Client Satisfaction Questionnaire [42]. This questionnaire includes eight items designed to assess various aspects of the patient's satisfaction with the treatment. Items are measured on a four-point scale. The sum score ranges from eight to 32 points.

Income and welfare benefits Income from regular employment and the duration of welfare benefits (for example, unemployment and sickness benefit) will be extracted from the GPI accounts.

\section{Therapeutic interventions}

Therapeutic interventions will be extracted from the standardized rehabilitation discharge letters [19]. These letters are stored in the individual GPI accounts. The documentation of the therapeutic interventions will indicate adherence to the WMR guideline.

\section{Department characteristics}

Measurement of department characteristics covers amongst others data on average guideline adherence and the number of treated patients. A survey of the departments will assess additional data on the department level (e.g. staff, team cooperation, and infrastructure).

\section{Other measures}

Additional measures will be assessed as potential effect modifiers and as variables for calculating the propensity score.

Risk of not returning to work Two risk scores are frequently used to assess the need for WMR in Germany. The score of the SIMBO (German: Screening-Instrument zur Feststellung des Bedarfs an medizinisch-beruflich orientierten Maßnahmen in der medizinischen Rehabilitation) [11] ranges from zero to 100 points. Higher scores indicate a higher risk of not returning to work and a stronger need for WMR. A SIMBO score of $\geq 23$ points was shown to be an optimal threshold to identify patients in need for WMR. The Würzburger Screening [12] states a risk of not returning to work and a need for WMR if a person is unemployed at the beginning of the rehabilitation program or scored one out of three points on a three-item scale that assesses negative return-to-work expectations.

Somatization Somatization will be assessed by using seven items from the Symptom Check-List-90-R [43]. All items are measured on a five-point scale $(0=$ not at all, 1 = a little bit, 2 = moderately, 3 = quite a bit, 4 = extremely). Items are averaged to calculate a total score.

Pain generalization Three newly developed items will assess the experience of widespread pain and pain amplification. These items are measured on a four-point scale $(0=$ totally disagree, $1=$ disagree, $2=$ agree, $3=$ totally agree). Items are averaged to calculate a total score.

Psychosocial stress Two items will assess family- and job-related stress in the last two weeks [44]. Both items are measured on a four-point scale $(0=$ not at all, $1=$ several days, $2=$ more than half of the days, $3=$ nearly all days). Items are added to a sum score ranging from zero to six points.

Work environment Several aspects of the work environment will be assessed as they might moderate the treatment effect: amongst others, size of company [45], physical job demands [46], effort-reward imbalance [47], overcommitment [47] and support by co-workers and supervisors.

Socio-demographic data We will ask participants for socio-demographic data (age, sex, native language, educational level, partnership, children, and family life).

\section{Statistical analysis \\ Propensity score matching}

Patients of WMR and CMR will be matched by propensity scores to achieve balanced sample characteristics and to calculate unbiased estimates of relative effectiveness $[22,48-54]$. The propensity score is the conditional probability of receiving the treatment under evaluation (i.e. WMR) given the vector of observed background variables. Matching by propensity scores enables balanced characteristics of the treated and the untreated sample if there is sufficient overlap of the propensity scores of both groups. Compared with a conventional direct matching procedure the problem of multidimensionality in finding a corresponding control, for instance related to age, sex, sickness absence duration, pain, depression and others, is thereby reduced to one dimension only.

The propensity score will be estimated by a logistic regression model including all variables that are associated 
with the treatment allocation. For every WMR participant a patient with a similar propensity score will be selected from the larger pool of CMR patients. Resampling will be realized without replacement. If necessary to achieve balanced data, a caliper of one-quarter of the propensity score during resampling will be used. For sensitivity analysis, additional matching schemes will be tested (with and without replacement, different calipers, varied number of controls). As an indicator of the bias before and after matching owed to differences related to the observed sample characteristics the standardized percentage bias will be calculated. This is the difference of the sample means in cases and controls relative to the square root of the average of the sample variances in both groups [55]. Multiple imputations will be used to fill in missing data before estimation of the propensity score. The propensity scores for each record will be averaged across the completed datasets, and propensity score matching will be performed with these averaged scores [56].

\section{Multilevel regression analyses}

Analyses of treatment effects in propensity score matched samples can use the same statistical methods as those used in experimental studies [48, 49]. Multilevel regression analyses will be used to account for dependencies in the data $[57,58]$. Individual patient data will be conceptualized as level-1 parameters and the rehabilitation department as a level-2 variable. All models will include the treatment variable (WMR vs. CMR), the baseline score of the respective outcome measure and a random intercept which reflects the rehabilitation department. For the primary outcomes, p-value will be fixed at 0.016 (Bonferroni corrected for three outcomes). For the secondary outcomes, $\mathrm{p}$-value will be fixed at 0.05 . Linear models will be used for continuous outcomes and logistic models for binary outcomes.

\section{Moderator analyses}

Exploratory moderator analyses will be performed to clarify whether level-1 or level-2 characteristics modify the treatment effect. The modeling of moderator effects of level-1 characteristics will be realized by multiplicative interaction terms of the treatment indicator and potential moderators. Moderator effects of level-2 variables will be tested by including the potential moderator as a level-2 predictor of the level-1 treatment-effect. In the case of continuous moderators, these variables will be $\mathrm{z}$ standardized [59]. Level-1 moderators will be tested in order to identify patients who particularly benefit from WMR. Level-2 moderators may suggest department characteristics that may impact the effectiveness of WMR.

\section{Discussion}

This study will provide an estimation of the relative effectiveness of a complex, newly implemented work-related rehabilitation strategy for patients with musculoskeletal disorders. Findings will complement the existing evidence of the relative efficacy derived from randomized controlled trials by robust estimation of the effects under real-life conditions of rehabilitation service provision in Germany. The findings of this study will be published in peerreviewed journal articles and conference presentations.

\section{Trial status}

Recruitment has started and is ongoing.

Abbreviations

CMR, conventional medical rehabilitation; GAD-2, Generalized Anxiety Disorder Questionnaire; GPI, German Pension Insurance; IRES-24, Indikatoren des Reha-Status; PHQ-2, Patient Health Questionnaire; WAI, Work Ability Index; WAS, Work Ability Score; WMR, work-related medical rehabilitation

\section{Acknowledgements \\ Not applicable.}

\section{Funding}

The study is funded by the Federal German Pension Insurance, Hohenzollerndamm 46/47, 10713 Berlin, Germany (grant number: FV-1253-15-0483-01). Funding covers personnel, material and traveling expenses. The Federal German Pension Insurance additionally contributes by sending out study materials and patient questionnaires and by providing administrative data on work participation and rehabilitation services of study participants. Anonymous data processing and data analyses are guaranteed.

\section{Availability of data and material}

Not applicable.

\section{Authors' contributions}

SN, MS, BS and MB developed the study design. All authors contributed to drafting the manuscript. All authors finally read and approved the manuscript for submission. All authors fulfill the authorship criteria of the International Committee of Medical Journal Editors.

\section{Competing interests}

The authors declare that they have no competing interests.

Consent for publication

Not applicable.

\section{Ethics approval and consent to participate}

The investigation conforms to the principles outlined in the Declaration of Helsinki. The study protocol was approved by the Ethics Committee of the University of Lübeck (15-356), and participation in the study is voluntary.

Eligible patients will receive written information on study aims, participation and the right to refuse. The study has been registered on the German Clinical Trials Register (DRKS00009780).

This paper contains the original study protocol. Any substantial modifications to the study protocol will be notified to the Ethics Committee of the University of Lübeck for approval prior to implementation. These amendments will be documented in detail in the German Clinical Trials Register and will be described transparently in trial reports.

\section{Author details}

${ }^{1}$ University of Applied Sciences Würzburg-Schweinfurt, Faculty of Applied Social Sciences, Münzstraße 12, Würzburg 97070, Germany. ${ }^{2}$ Institute of Social Medicine and Epidemiology, University of Lübeck, Ratzeburger Allee 160, Lübeck 23562, Germany. ${ }^{3}$ Department of Medical Psychology and Psychotherapy, Medical Sociology and Rehabilitation Sciences, University of Würzburg, Klinikstraße 3, Würzburg 97070, Germany. 
Received: 16 July 2016 Accepted: 3 August 2016 Published online: 17 August 2016

\section{References}

1. Eurostat. Disability statistics - prevalence and demographics. 2016. http://ec.europa.eu/eurostat/statistics-explained/index.php/Disability_ statistics_-_prevalence_and_demographics. Accessed 10 Aug 2016.

2. Loisel P, Buchbinder R, Hazard R, Keller R, Scheel I, van Tulder M, Webster B. Prevention of work disability due to musculoskeletal disorders: the challenge of implementing evidence. J Occup Rehabil. 2005;15:507-24.

3. Schaafsma FG, Whelan K, van der Beek AJ, van der Es-Lambeek LC, Ojajarvi A, Verbeek JH. Physical conditioning as part of a return to work strategy to reduce sickness absence for workers with back pain. Cochrane Database Syst Rev. 2013;8:Cd001822.

4. Kamper SJ, Apeldoorn AT, Chiarotto A, Smeets RJ, Ostelo RW, Guzman J, van Tulder MW. Multidisciplinary biopsychosocial rehabilitation for chronic low back pain. Cochrane Database Syst Rev. 2014:9:Cd000963.

5. van Vilsteren $\mathrm{M}$, van Oostrom $\mathrm{SH}$, de Vet HC, Franche RL, Boot CR, Anema JR. Workplace interventions to prevent work disability in workers on sick leave. Cochrane Database Syst Rev. 2015;10:Cd006955.

6. Brüggemann S. The Rehabilitation Guideline Program of the BfA. Z Arztl Fortbild Qualitatssich. 2005:99:47-50.

7. Hüppe A, Raspe H. Efficacy of inpatient rehabilitation for chronic back pain in Germany: a systematic review 1980-2001. Rehabilitation. 2003:42:143-54.

8. Hüppe A, Raspe H. Efficacy of inpatient rehabilitation for chronic back pain in Germany: update of a systematic review. Rehabilitation. 2005;44:24-33.

9. Hüppe A, Glaser-Möller N, Raspe H. Offering multidisciplinary medical rehabilitation to workers with work disability due to musculoskeletal disorders: results of randomized controlled trial. Gesundheitswesen. 2006;68:347-56

10. Bethge M. Success factors of work-related orthopaedic rehabilitation. Rehabilitation. 2011;50:145-51.

11. Streibelt M, Bethge M. Prospective cohort analysis of the predictive validity of a screening instrument for severe restrictions of work ability in patients with musculoskeletal disorders. Am J Phys Med Rehabil. 2015:94:617-26.

12. Lukasczik M, Wolf HD, Gerlich C, Löffler S, Vogel H, Faller H, Neuderth S. Current state of vocationally oriented medical rehabilitation - a German perspective. Disabil Rehabil. 2011;33:2646-55

13. Streibelt M, Buschmann-Steinhage R. A profile of requirements for the performance of work related medical rehabilitation from the perspective of the statutory pension insurance. Rehabilitation. 2011:50:160-7.

14. Bethge M, Herbold D, Trowitzsch L, Jacobi C. Work status and health-related quality of life following multimodal work hardening: a cluster randomised trial. J Back Musculoskelet Rehabil. 2011;24:161-72.

15. Kleist B, Alliger K, Winter S, Beyer WF. Work Hardening bei chronischen unspezifischen Rückenschmerzen in der stationären Rehabilitation. Praxis Klin Verhaltensmed Rehab. 2001;14:145-50.

16. Streibelt M, Bethge M. Effects of intensified work-related multidisciplinary rehabilitation on occupational participation: a randomized-controlled trial in patients with chronic musculoskeletal disorders. Int J Rehabil Res. 2014:37:61-6.

17. Bethge $M$, Neuderth S. Medizinisch-berufliche Maßnahmen. In: Bengel J Mittag O, editors. Psychologie in der medizinischen Rehabilitation. Ein Lehr- und Praxishandbuch. Heidelberg: Springer; 2016.

18. Deutsche Rentenversicherung Bund. Anforderungsprofil zur Durchführung der Medizinisch-beruflich orientierten Rehabilitation (MBOR) im Auftrag der Deutschen Rentenversicherung. 3., überarbeitete Auflage. Berlin: Deutsche Rentenversicherung Bund; 2012.

19. Bethge M, Löffler S, Schwarz B, Vogel H, Schwarze M, Neuderth S. Is the guideline for work-related medical rehabilitation successfully implemented? Rehabilitation. 2014;53:184-90.

20. Bethge M, Löffler S, Schwarz B, Vogel H, Schwarze M, Neuderth S. Specific work-related problems: do they matter in access to work-related medical rehabilitation? Rehabilitation. 2014:53:49-55.

21. Glasgow RE, Lichtenstein E, Marcus AC. Why don't we see more translation of health promotion research to practice? Rethinking the efficacy-to-effectiveness transition. Am J Public Health. 2003;93:1261-7.

22. Schelvis RM, Oude Hengel KM, Burdorf A, Blatter BM, Strijk JE, van der Beek AJ. Evaluation of occupational health interventions using a randomized controlled trial: challenges and alternative research designs. Scand J Work Environ Health. 2015;41:491-503.
23. Chan AW, Tetzlaff JM, Gotzsche PC, Altman DG, Mann H, Berlin JA, Dickersin K, Hrobjartsson A, Schulz KF, Parulekar WR, et al. SPIRIT 2013 explanation and elaboration: guidance for protocols of clinical trials. BMJ. 2013;346:e7586.

24. Zoer I, de Graaf L, Kuijer PP, Prinzie P, Hoozemans MJ, Frings-Dresen MH. Matching work capacities and demands at job placement in employees with disabilities. Work. 2012;42:205-14.

25. Toeppen-Sprigg B. Importance of job analysis with functional capacity matching in medical case management: a physician's perspective. Work. 2000;15:133-7.

26. Voss M, Schutte M, Wieland K. Synopsis of procedures used in the evaluation of the physically handicapped and work demands. Rehabilitation. 1988;27:135-9.

27. Kuijer PP, Gouttebarge V, Wind H, van Duivenbooden C, Sluiter JK, Frings-Dresen $\mathrm{MH}$. Prognostic value of self-reported work ability and performance-based lifting tests for sustainable return to work among construction workers. Scand J Work Environ Health. 2012;38:600-3.

28. IImarinen J. The Work Ability Index (WAI). Occup Med (Lond). 2007:57:160.

29. El Fassi M, Bocquet V, Majery N, Lair ML, Couffignal S, Mairiaux P. Work ability assessment in a worker population: comparison and determinants of Work Ability Index and Work Ability Score. BMC Public Health. 2013;13:305.

30. Kristensen TS, Hannerz H, Hogh A, Borg V. The Copenhagen Psychosocial Questionnaire-a tool for the assessment and improvement of the psychosocia work environment. Scand J Work Environ Health. 2005;31:438-49.

31. Nuebling M, Hasselhorn HM. The Copenhagen Psychosocial Questionnaire in Germany: from the validation of the instrument to the formation of a job-specific database of psychosocial factors at work. Scand J Public Health. 2010;38:120-4.

32. Wirtz M, Farin E, Bengel J, Jäckel WH, Hammerer D, Gerdes N. IRES-24 patient questionnaire: development of the short form of an assessment instrument in rehabilitation by means Mixed-Rasch analysis. Diagnostica. 2005:51:75-87.

33. Lowe B, Kroenke K, Grafe K. Detecting and monitoring depression with a two-item questionnaire (PHQ-2). J Psychosom Res. 2005;58:163-71.

34. Kroenke K, Spitzer RL, Williams JB, Lowe B. The Patient Health Questionnaire Somatic, Anxiety, and Depressive Symptom Scales: a systematic review. Gen Hosp Psychiatry. 2010:32:345-59.

35. Von Korff M, Ormel J, Keefe FJ, Dworkin SF. Grading the severity of chronic pain. Pain. 1992:50:133-49.

36. Klasen BW, Hallner D, Schaub C, Willburger R, Hasenbring M. Validation and reliability of the German version of the Chronic Pain Grade questionnaire in primary care back pain patients. Psychosoc Med. 2004;1:Doc07.

37. Pfingsten $\mathrm{M}$, Kroner-Herwig $\mathrm{B}$, Leibing $\mathrm{E}$, Kronshage $\mathrm{U}$, Hildebrandt J. Validation of the German version of the Fear-Avoidance Beliefs Questionnaire (FABQ). Eur J Pain. 2000;4:259-66.

38. Kent P, Mirkhil S, Keating J, Buchbinder R, Manniche C, Albert HB. The concurrent validity of brief screening questions for anxiety, depression, social isolation, catastrophization, and fear of movement in people with low back pain. Clin J Pain. 2014;30:479-89.

39. Godin G, Shephard RJ. A simple method to assess exercise behavior in the community. Can J Appl Sport Sci. 1985;10:141-6.

40. Osborne RH, Elsworth GR, Whitfield K. The Health Education Impact Questionnaire (heiQ): an outcomes and evaluation measure for patient education and self-management interventions for people with chronic conditions. Patient Educ Couns. 2007;66:192-201.

41. Mittag O, Raspe H. A brief scale for measuring subjective prognosis of gainful employment: findings of a study of 4279 statutory pension insurees concerning reliability (Guttman Scaling) and validity of the scale. Rehabilitation. 2003;42:169-74.

42. Attkisson CC, Zwick R. The client satisfaction questionnaire. Psychometric properties and correlations with service utilization and psychotherapy outcome. Eval Program Plann. 1982:5:233-7.

43. Schmitz N, Hartkamp N, Kiuse J, Franke GH, Reister G, Tress W. The Symptom Check-List-90-R (SCL-90-R): a German validation study. Qual Life Res. 2000:9:185-93.

44. Küch D, Arndt S, Grabe A, Manthey W, Schwabe M, Fischer D. UKS Ultrakurzscreening psychosozialer Problemlagen zur bedarfsorientierten Angebotszuweisung in der somatischen Rehabilitation. In: Arbeitskreis Klinische Psychologie in der Rehabilitation Fachgruppe der Sektion Klinische Psychologie im Berufsverband Deutscher Psychologinnen und Psychologen (BDP) e. V., editors. Psychologische Betreuung im Krankheitsverlauf. Berlin: Deutscher Psychologen Verlag; 2011. p. 106. 
45. European Commission. User guide to the SME definition. Luxembourg: Publications Office of the European Union; 2015.

46. Bethge M, Spanier K, Neugebauer T, Mohnberg I, Radoschewski FM. Self-reported poor work ability-an indicator of need for rehabilitation? A cross-sectional study of a sample of German employees. Am J Phys Med Rehabil. 2015;94:958-66.

47. Siegrist J, Wege N, Puhlhofer F, Wahrendorf M. A short generic measure of work stress in the era of globalization: effort-reward imbalance. Int Arch Occup Environ Health. 2009:82:1005-13.

48. Guo S, Fraser MW. Propensity score analysis: statistical methods and applications. Los Angeles: Sage Publications; 2010.

49. Rosenbaum PR, Rubin DB. The central role of the propensity score in observational studies for causal effects. Biometrika. 1983;70:41-55.

50. Dehejia RH, Wahba S. Propensity score-matching methods for nonexperimental causal studies. Rev Econ Stat. 2002;84:151-61.

51. Descatha A, Leclerc A, Herquelot E. Use of propensity scores in occupational health? J Occup Environ Med. 2013:55:477-8.

52. Saltychev M, Laimi K, El-Metwally A, Oksanen T, Pentti J, Virtanen M, Kivimaki M, Vahtera J. Effectiveness of multidisciplinary primary prevention in decreasing the risk of work disability in a low-risk population. Scand J Work Environ Health. 2012;38:27-37.

53. Saltychev M, Laimi K, Oksanen T, Pentti J, Kivimaki M, Vahtera J. Does perceived work ability improve after a multidisciplinary preventive program in a population with no severe medical problems - the Finnish Public Sector Study. Scand J Work Environ Health. 2013;39:57-65.

54. Bethge M. Effects of graded return-to-work: a propensity-score-matched analysis. Scand J Work Environ Health. 2016;42:273-9.

55. Rosenbaum PR, Rubin DB. Constructing a control group using multivariate matched sampling methods that incorporate the propensity score. Am Stat. 1985;39:33-8.

56. Mitra R, Reiter JP. A comparison of two methods of estimating propensity scores after multiple imputation. Stat Methods Med Res. 2016;25:188-204.

57. Kreft I, Leeuw J. Introducing multilevel modeling. London: Sage; 2006.

58. Donner A, Klar N. Design and analysis of cluster randomization trials in health research. London: Arnold; 2000.

59. Smeets RJ, Maher CG, Nicholas MK, Refshauge KM, Herbert RD. Do psychological characteristics predict response to exercise and advice for subacute low back pain? Arthritis Rheum. 2009;61:1202-9.

\section{Submit your next manuscript to BioMed Central and we will help you at every step:}

- We accept pre-submission inquiries

- Our selector tool helps you to find the most relevant journal

- We provide round the clock customer support

- Convenient online submission

- Thorough peer review

- Inclusion in PubMed and all major indexing services

- Maximum visibility for your research

Submit your manuscript at www.biomedcentral.com/submit 